\title{
How much is enough: 3 versus 6 months of adjuvant therapy for colon cancer
}

\author{
John L Marshall*,1 \\ ${ }^{1}$ Ruesch Center, Lombardi, Georgetown University, Washington, DC 20007, USA \\ *Author for correspondence: marshalj@georgetown.edu
}

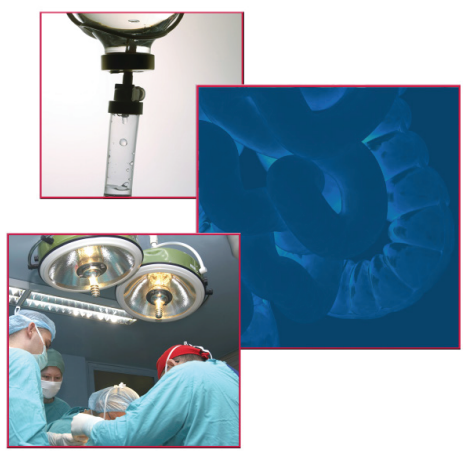

\section{"we also must acknowledge our virtually indiscriminate use of adjuvant therapy as one of our great failures"}

First draft submitted: 27 October 2017; Accepted for publication: 9 November 2017; Published online: 30 January 2018

Keywords: adjuvant $\bullet$ colon $\bullet$ duration

\section{The evolution of adjuvant therapy in stage III colon cancer Important principles}

There is no question that adjuvant chemotherapy is one of the great successes in oncology. The reality that treating someone with systemic therapy in conjunction with a surgical resection of the primary tumor increases the cure rate drives us all to identify and treat all appropriate patients to ensure that no single patient relapses and dies of their cancer if it could have been avoided. As the surgeon is 'pulling the weed' by the roots, we are 'treating the yard' to ensure that no subsequent metastasis (seeds) ever grow. Our successes in adjuvant therapy across all tumor types have contributed significantly to our growing number of cancer survivors around the world.

However, we also must acknowledge our virtually indiscriminate use of adjuvant therapy as one of our great failures - we are terrible at patient selection. We expose large populations of patients to toxic agents, some of whom do not need treatment because there was no microscopic metastatic disease (the surgeon really did 'get it all') and some who do have microscopic metastatic disease but do not benefit from the therapy (we assume either resistant to the therapy or the metastases are beyond cure - the seeds have grown roots). Our reality is that in virtually every disease type, there is only a relatively small subset of patients who benefit from adjuvant therapy and are subsequently converted to 'cured' by adjuvant treatment. Everyone experiences toxicity, everyone experiences anxiety, only a few are positively affected. While we are working toward the development of biomarkers that will predict who should get therapy and who should not, we are still in an era of empiric decision-making. Our desire to cure every last patient possible is stronger than our respect for the negative impact of the treatment itself. Patients are appropriately afraid of a relapse, and will do almost anything to avoid it. Our culture equates cancer treatment with war, a battle, struggle or fight. There is an expectation on their part that cure requires suffering. A cancer diagnosis comes with the expectation of sacrifice.

Until we have better biomarkers for patient selection, this is our current standard. Given this understandable predicament, it is incumbent upon us to both optimize the effectiveness of the treatment and minimize the toxicity. Let us briefly review the history of adjuvant therapy for stage III colon cancer. I have been around long enough to remember the day when adjuvant therapy for colon cancer involved 18 months of intravenous fluorouracil (5-FU). Without any trial evidence, we then moved to 12 months of treatment. [1,2]. Finally, a clinical trial was done comparing 6-12 months of intravenous 5-FU with 6 months showing similar benefit [3], but please note, was not a very large trial and not a noninferiority design. So in point of fact, we do not know if some patients may have benefitted from 12 months. Given the relative ease and relatively low toxicity profile of intravenous 5-FU, 6 months of treatment has long been our standard with no compelling need to shorten the duration. Then, oxaliplatin came along. 


\section{Oxaliplatin neurotoxicity}

Randomized clinical trials clearly demonstrated that the addition of oxaliplatin to intravenous 5-FU improved the survival rate of patients with stage III colon cancer [4]. While the absolute magnitude of benefit was small, in the range of 3-5\%, these were three-to-five patients out of 100 who would be cured of their disease. We were also in an era when dose intensity was an important concept and thought to be critical for all cancers. Finally, we were led to believe that the cumulative neurotoxicity of oxaliplatin would reverse with time [5]. Therefore, it was critical to push through to the end of 12 cycles (6 months) of adjuvant 5-FU and oxaliplatin, almost despite toxicity. This was true for stage II, III, IV no evidence of disease, rectal - everyone. The mantra was "better to be alive with neuropathy than not alive". Our enthusiasm for breakthroughs and more patients being cured were enough for us to justify pressing all patients regardless of their individual benefit to the end.

Only years later did we begin to lose our enthusiasm. We are all now monitoring a large (thankfully) patient population with significant life-altering neuropathy. Increasingly, it became clear that we needed to reevaluate the role that oxaliplatin plays - specifically, could we maintain the survival benefit offered by the addition of oxaliplatin while minimizing the toxicity by shortening the overall duration of therapy. In breast cancer and other diseases, large randomized trials have been performed which demonstrated that shorter duration of chemotherapy was just as effective as longer durations, and so it seemed prudent for us to study the same question in colorectal cancer.

\section{IDEA results}

Thus, the IDEA trial was conceived and carried out - a global feat of which we should all be proud. To clearly demonstrate that there was no loss of effectiveness, six large randomized clinical trials done globally were prospectively planned to be analyzed together to answer the question of the noninferiority of 3 months of oxaliplatin-based chemotherapy compared with 6 months [1]. This study was presented initially at the 2017 ASCO meeting [6] and, in my opinion, represents a landmark moment for us and for our patients.

The full manuscript has not been published but the data presented in abstract form demonstrated that patients with lower-risk stage III colon cancers (T1-3N1) faired just as well with 3 months of therapy and have significantly reduced neurotoxicity compared with those who get 6 months of therapy. This group met the end point of noninferiority. Second, those with a higher-risk stage III colon cancer (T4N2+) do virtually as well but did not meet the noninferiority statistical bar. Out of every 100 patients treated, 38 versus 36 patients will relapse if only 3 months of chemotherapy is given. Third, and maybe most intriguing, capecitabine (CAPEOX) appears to perform better than iv. 5-FU (FOLFOX). Each of these findings is immediately practice changing. By selecting patients who can be given only 3 months of treatment and not lose any survival, we have reduced costs, toxicity and improved survivorship for many. For those where 6 moths may be of benefit, the results give us the clearest dataset to date to discuss with our patients. They can now be more informed participants in the risk-benefit discussion.

\section{Discussion}

The IDEA trial gives us immediate permission to give patients less chemotherapy in the stage III adjuvant setting. Supported by these data and my experience, my practice is to offer capecitabine and oxaliplatin for 3 months to all patients (under the age of 70) with a re-evaluation to determine whether or not chemotherapy should be continued in those patients with higher-risk stage III disease. Virtually never do I go beyond eight cycles of oxaliplatin, even in those patients with high risk. In my opinion, the IDEA study gives us permission to stop oxaliplatin at 3 months - in higher-risk, we should discuss whether or not we continue the 5-FU/capecitabine for a total of 6 months.

For final recommendations, we need these data to mature to answer the question - does the 3-year disease-free survival end point translate into overall survival? In the meantime, we need to note that many of our patients suffer with significant neuropathy and other postadjuvant toxicities that significantly alter their quality of life. Our enthusiasm to cure every single patient possible must not be dampened. But we must use these data to improve our patient selection empowering us to avoid cumulative toxicity.

Our patients want to be 'aggressive'. We want to be effective. I have had several patients since these data have emerged who are uncertain about stopping at 3 months. I have shown the actual (easy to read) slides from the ASCO presentation to patients to help in the decision-making. We and our patients do not want to look back with regret, wondering that if only they had received more chemotherapy, their cancer would have never come back. I believe the IDEA trial gives us very solid ground to say that even across the board very few patients benefit from the second half of a 6 -month adjuvant treatment course. 
Maybe more problematic for the US oncologist, do we have to change to capecitabine? There is so much that we do not know about the adjuvant treatment of colon cancer. Our drugs that work well in the metastatic setting do not work in the adjuvant setting. We are not sure of the target cell itself, is it an epithelial cell, a mesenchymal cell or some other cell type? Does a more continuous exposure of 5-FU from capecitabine offer more benefit in attacking the target cells? Finally, these results immediately begin the debate on how to apply them to stage II disease, to rectal cancer or even to perioperative stage IV no evidence of disease patients. I am certain that we do not want to spend the time or enormous number of patients that would be required to answer these specific questions. Instead, as we did at the beginning of our story, we will have to decide as a community and declare by convention, whether 3 months of adjuvant therapy is appropriate in these clinical scenarios. I would recommend that we do and move on from there, to find the true biomarkers we seek.

\section{Financial \& competing interests disclosure}

The author has no relevant affiliations or financial involvement with any organization or entity with a financial interest in or financial conflict with the subject matter or materials discussed in the manuscript. This includes employment, consultancies, honoraria, stock ownership or options, expert testimony, grants or patents received or pending, or royalties.

No writing assistance was utilized in the production of this manuscript.

\section{References}

1. Andre T, Iveson T, Labianca R et al. The IDEA (International Duration Evaluation of Adjuvant Chemotherapy) collaboration: prospective combined analysis of Phase III trials investigating duration of adjuvant therapy with the FOLFOX (FOLFOX4 or modified FOLFOX6) or XELOX (3 versus 6 months) regimen for patients with stage III colon cancer: trial design and current status. Curr. Colorectal Cancer Rep. 9, 261-269 (2013).

2. Moertel CG, Fleming TR, Macdonald JS et al. Levamisole and fluorouracil for adjuvant therapy of resected colon carcinoma. N. Engl. J. Med. 322(6), 352-358 (1990).

3. O'connell MJ, Laurie JA, Kahn M et al. Prospectively randomized trial of postoperative adjuvant chemotherapy in patients with high-risk colon cancer. J. Clin. Oncol. 16(1), 295-300 (1998).

4. Andre T, Boni C, Mounedji-Boudiaf L et al. Oxaliplatin, fluorouracil, and leucovorin as adjuvant treatment for colon cancer. $N$. Engl. J. Med. 350(23), 2343-2351 (2004).

5. Grothey A. Oxaliplatin-safety profile: neurotoxicity. Semin. Oncol. 30(4 Suppl. 15), 5-13 (2003).

6. Shi Q, Sobrero AF, Shields AF et al. Prospective pooled analysis of six Phase III trials investigating duration of adjuvant (adjuv) oxaliplatin-based therapy (3 vs 6 months) for patients (pts) with stage III colon cancer (CC): The IDEA (International Duration Evaluation of Adjuvant chemotherapy) collaboration. J. Clin. Oncol. 35 (18 Suppl.), LBA1-LBA1 (2017). 